\title{
El puerto y la vinculación entre lo local y lo global
}

\author{
Carlos Martner Peyrelongue*
}

\begin{abstract}
Ports used to be spaces of little interest during the "inwards" development model based on imports substitution. Hence, the indifference for its study in the diverse fields of social science, even in areas too close to this topic, as regional analysis and economical geography. However, due to the recent restructure of global capitalism system, these coast line spaces have got more importance at establishing themselves as main hubs in the articulation of productive networks recently globalized. Precisely, this article intends to analyze space and temporal implications of this recent rol of ports in the articulation of extended production-distribution networks, which redefines the links between local and global, but at a time they originate wide exclusion spaces. Here, concepts as "flow space", "network territory" and "simultaneous space-time" of globalization, that rescue from the regional analysis and the economical geography, the systemic-structural tradition developed in the social sciences, mainly by Braudel and Wallerstein, result important by its explanatory force in times of a firm restructure of the seaport space and generally of the capital world-economics.
\end{abstract}

Key words: Port, hinterland, network, hub, space of flows, global and local.

\section{Resumen}

Los puertos fueron espacios poco relevantes durante el periodo en que privó el modelo de desarrollo "hacia adentro" basado en la sustitución de importaciones. De allí, el desinterés por su estudio dentro de los distintos campos de las ciencias sociales, inclusive en áreas muy cercanas con esta temática, como el análisis regional y la geografía económica. Sin embargo, a raíz de la reciente reestructuración del sistema mundial capitalista, estos espacios litorales han adquirido mayor relevancia al constituirse en nodos principales de articulación de redes productivas crecientemente globalizadas. Precisamente, en este artículo se pretende hacer un análisis de las implicaciones espaciales y temporales del rol recientemente asumido por los puertos en la articulación de extensas redes de producción-distribución, las cuales redefinen los vínculos entre lo local y lo global, pero a la vez generan vastos espacios de exclusión. Aquí, los conceptos como "espacio de flujos", "territorio de redes" y " el espacio-tiempo simultáneo” de la globalización, que rescatan, desde el análisis regional y la geografía económica, la tradición estructural-sistémica desarrollada en las ciencias sociales principalmente por Braudel y Wallerstein, resultan relevantes por su fuerza explicativa en momentos de fuerte reestructuración del ámbito portuario y, en general, de la economía-mundo capitalista.

Palabras Claves: Puerto, hinterland, redes, nodos, espacio de flujos, global y local.

\footnotetext{
* Doctor en Ciencias Sociales. Investigador del Instituto Mexicano del Transporte. Profesor de Distribución Física Internacional en la Universidad Autónoma de Querétaro. Apartado Postal 1098, C.P. 76000 Querétaro, Gro. México. E-mail: Carlos.Martner@imt.mx
}

Revista eure (Vol. XXV, N 75), pp. 103-120, Santiago de Chile, septiembre 1999 
Carlos Martner Peyrelongue

\section{INTRODUCCIÓN}

$\mathrm{L}$ os puertos se han caracterizado por ser lugares claves de intercambio de mercancías y, a la vez, lugares de vínculo entre culturas y personas. El puerto es frontera geográfica, tecnológica y cultural, por eso es un punto de ruptura, pero, a la vez, es lugar de encuentro, es intersección de rutas comerciales y zona de confluencia de ideas, valores y tecnologías de origen diverso. Sin embargo, es difícil encontrar una caracterización perenne de los puertos y de su relación con el territorio. Las características y funciones de los puertos evolucionan en la medida en que las formaciones económico-sociales y las regulaciones interiores de los países se modifican.

En el contexto actual de configuración de redes extensas que articulan regiones selectas del planeta, los puertos, como espacios de frontera, de vínculo entre lo interno y lo externo, entre lo local y lo global, adquieren un papel relevante en la reestructuración territorial en curso. Por esta razón, en este artículo se pretende hacer una reflexión sobre las connotaciones territoriales y espaciotemporales de la integración de los puertos en la fase actual de desarrollo de la economía-mundo capitalista (Wallerstein, 1990), caracterizada entre otras cosas por procesos de apertura comercial generalizados, desregulación y privatización de las actividades económicas y una posición más débil del sistema interestatal (Wallerstein, 1996) frente a la formación de redes de producción-distribución globales, comandadas principalmente por grandes consorcios transnacionales y articuladas en un "espacio de flujos" (Castells, 1996), cuyo ámbito de acción se sobrepone y rebasa constantemente las fronteras nacionales y las actuaciones estatales.
En este sentido, la unidad de análisis de los estudios sobre los puertos y sus vínculos territoriales difícilmente podrá ubicarse en las sociedades locales o nacionales, por el contrario, para comprender las dinámicas actuales en esta temática parece más adecuado el enfoque braudeliano o wallersteriano que ubica la unidad de análisis en los avatares y transformaciones del sistema mundial y la economía-mundo capitalista. Dentro del análisis regional, resultan relevantes los conceptos de autores que retoman este enfoque estructuralsistémico, como Manuel Castells (1996), con su planteamiento del "espacio de flujos" global, y Pierre Veltz (1994) con la conceptualización del territorio-red que se sobrepone a los territorios-zona o regiones continuas convencionales, entre otros. Aquí interesa, precisamente, retomar esta escuela de pensamiento para analizar una temática muy poco expuesta dentro de las ciencias sociales $\mathrm{y}$, en particular, dentro de los estudios regionales y la geografía económica, como es el caso del establecimiento del rol que adquieren los puertos tanto en la reestructuración territorial en curso como en la articulación espacio-temporal de redes que vinculan ámbitos territoriales diversos y distantes.

En primera instancia, se analizarán, desde la perspectiva de las formas de inserción y articulación territorial, las diferencias esenciales entre los puertos del periodo de sustitución de importaciones, caracterizado por la protección de las economías nacionales y el fortalecimiento del mercado interno, y los de la actual fase de liberalización, apertura comercial y fragmentación global de la producción. Posteriormente, se profundiza sobre el impacto de los cambios espacio-temporales recientes en los ámbitos portuarios y sobre las condicionantes que impone la formación de redes globales en su desarrollo. Inelu- 
El puerto y la vinculación entre lo local $\bar{y}$ lo global

diblemente, aquí aparece con peculiar significación el tema de la transformación del puerto en un nodo de articulación entre los ámbitos locales y el ámbito global. Finalmente se concluye realizando una reflexión, seguramente todavía incompleta, sobre el papel de los puertos en una forma de organización territorial emergente, estructurada por redes y flujos.

\section{Del hinterland CAUTIVO AL HINTERLAND COMÚN}

\section{A. La zona de influencia del puerto del proteccionismo}

En épocas en que privó el proteccionismo, los puertos redujeron su actividad y debilitaron sus contactos. En efecto, el puerto del modelo de desarrollo latinoamericano basado en la industrialización por sustitución de importaciones debilitó su posición como lugar de enlace internacional, como punto de contacto entre lo interno y lo externo, como lugar de intercambio mercantil y cultural.

En esa fase, el puerto estableció limitados vínculos con el exterior, en buena medida, se cerró a su "foreland", o sea, a su zona de influencia externa y, al proceder de esta forma, debilitó simultáneamente su "hinterland" ${ }^{1}$ o zona de influencia interna. Así, se convirtió en puerta de difícil acceso, en frontera rígida. En este sentido, el puerto perdió, en parte, el ambiente cosmopolita de intercambio de productos y de ideas que lo

\footnotetext{
${ }^{1}$ Bird (1971), en su análisis geográfico de los puertos, señala que éstos se establecen en una zona intermedia, como una "puerta de salida" (gateway) ubicada entre el hinterland o zona de influencia de tierra adentro y el foreland o zona de influencia externa, de ultramar.
}

caracterizó en otras épocas, en especial, en el siglo XIX, donde la expansión capitalista apoyada en importantes innovaciones tecnológicas vinculó gran cantidad de países y regiones.

Los desplazamientos del puerto hacia el interior y el exterior fueron escasos y lentos durante este periodo. Se destacó la característica de lugar de ruptura de carga y de tracción, al tiempo que se debilitó su función como lugar de enlace entre mundos distintos. En otras palabras, sobresalió el carácter de lugar donde la mercancía termina o inicia un recorrido en un modo de transporte específico, en un barco. El puerto era concebido como punto terminal donde las mercancías permanecían largo tiempo estacionadas en un lento recorrido hasta el destino final. Primó entonces el carácter de estación terminal, de ruptura larga, de zona de almacenamiento prolongado, de bodega. En muchos sentidos las zonas costeras aparecían desvinculadas de las regiones interiores y, por eso, más que lugares de vinculación eran puntos de quiebre, de separación de la influencia externa.

De esta forma, el puerto perdió importancia. Su actividad como lugar de intersección de rutas se debilitó, los flujos se "adelgazaron" y los contactos fueron reducidos. Sólo aquellos puertos que exportaban materias primas tuvieron un mayor dinamismo, aunque muchas veces se constituyeron como enclaves, con pocas conexiones con las regiones interiores del país. La debilidad de los enlaces terrestres entre la costa y el interior hasta bien entrado el siglo Xx es muestra de este proceso.

En lo político se reforzaron las tendencias aislacionistas/proteccionistas de los puertos, generando una legislación que no sólo limitó el movimiento de mu- 
Carlos Martner Peyrelongue

chas mercancías, sino que también puso controles y trámites que eternizaban los desplazamientos a través del puerto. Todos éstos son elementos que contribuyeron a la desvalorización del puerto como lugar, como espacio vinculado.

Tal como señala el Grupo Intergubernamental de Expertos en Puertos de la UNCTAD, "los puertos no desempeñaban un papel importante en la economía de un país cuando el desarrollo de está dependía sobre todo de los mercados nacionales". (Unctad, 1992, p. 15). Los puertos establecían vínculos comerciales y territoriales con su región inmediata, pero difícilmente podían tener influencia sobre procesos productivos y comerciales de regiones interiores más alejadas. Esta tendencia se vio reforzada por la falta de integración de los sistemas de transporte, lo cual impedía establecer redes continuas de flujos "puerta a puerta".

Así, el "hinterland" de los puertos era restringido, pero además era prácticamente cautivo, dadas las dificultades de accesibilidad. Tal como se señala en el informe de Expertos en Puertos, "antes cada puerto solía tener su propio grupo de clientes, cuyas actividades estaban situadas en las cercanías de la zona portuaria $y$ de su hinterland cautivo, muchas veces fuera del alcance de otros puertos debido a un sistema de transporte terrestre subdesarrollado y oneroso y a veces a obstáculos politicos y administrativos" (Unctad, 1992, p. 40). Tal situación otorgaba a los puertos cierta estabilidad y una relativa importancia con el ámbito regional y local más próximo. Así, más que una articulación territorial amplia de tipo red, se daba una vinculación con el área inmediata, es decir, de tipo zona.

Ahora bien, con la crisis del modelo de sustitución de importaciones y del proyecto desarrollista en América Latina, así como con el agotamiento, a nivel mundial, del régimen de acumulación fordista, la situación de los puertos y sus vinculaciones territoriales ha tendido a modificarse. $^{2}$

La reestructuración de la economíamundo capitalista (Wallerstein, 1996) y la búsqueda de un nuevo modelo de acumulación, apoyado en los recientes avances científico-técnicos, han propiciado la fragmentación de la producción a nivel mundial (o continental, al menos) diversificando la localización de las etapas que integran el proceso productivo, con la finalidad de encontrar ventajas para cada una de ellas. Esta vía, complementada por la apertura comercial y la desregulación económica de los países periféricos, otorgan al puerto nuevas características.

\section{B. El hinterland del puerto de la globalización}

En esta etapa de apertura comercial y de globalización de las relaciones económicas, el puerto no deja de caracterizarse por la ruptura de tracción, dado el necesario cambio de modo de transporte que se realiza en este lugar; sin embargo, la creciente integración de los sistemas de transporte ha eliminado la ruptura de carga, creando la posibilidad de que las terminales marítimas se conviertan principalmente en nodos de transferencia de flujos hacia y desde las regiones interiores. El puerto de la apertura y la globalización revaloriza su localización territorial, en tanto fortalece

2 El llamado régimen de acumulación fordista se caracterizó, entre otras cosas, por la producción industrial masiva en las lineas de montaje, por el aumento de la productividad en proporción similar a la de los ingresos reales, por la ampliación de los mercados, por una fuerte expansión mundial del capital y por la participación del Estado en la economía y en el desarrollo de instituciones de bienestar social. 
El puerto y la vinculación entre lo local y lo global

su posición como nodo de enlace, como lugar de encuentro y de articulación entre espacios del interior y del exterior.

A diferencia del periodo proteccionista, donde el puerto resaltó su condición de borde, de valla de contención frente al exterior, ahora se revaloriza como espacio vinculante e interactivo, principalmente entre el ámbito local y el global. Por eso, el puerto de la globalización no es terminal, sino "hub" (Fossey, 1997) o centro de procesamiento, distribución e integración de cadenas productivas fragmentadas internacionalmente. Pero lo que ha permitido la acción integradora del puerto actual, así como la vasta expansión de su zona de influencia territorial (hinterland), es, básicamente, el desarrollo de los sistemas intermodales de transporte e información.

El intermodalismo en su definición más general tiene que ver con la integración de los diversos modos de transporte en una sola red de distribución física de las mercancías. En otras palabras, los modos de transporte marítimo y terrestre (y en ocasiones el aéreo) son coordinados e integrados en un solo sistema o red que busca eliminar las rupturas de carga para que los productos fluyan entre lugares distantes en el menor tiempo posible y con la mayor certeza (Martner, 1998).

Un elemento clave en el desarrollo del intermodalismo, que precisamente procede del transporte marítimo y los puertos, ha sido la invención del contenedor. Esta es por excelencia la tecnología que permite integrar las redes de transporte terrestre con las redes maritimas. En la medida en que puede ser movido indistintamente por un camión, un ferrocarril o un barco, evita las rupturas de carga en las terminales portuarias o interiores. Tales características tecnológicas permiten acelerar los flujos de mer- cancías e integrar efectivamente procesos productivos fragmentados en el espacio. En esa medida permite articular los puertos con regiones distantes del interior (Martner, 1998).

Antes del desarrollo del intermodalismo era virtualmente imposible desarrollar una red global de transportes. En efecto, la separación de los modos de transporte impedía el desarrollo de los flujos "puerta a puerta", es decir, desde la puerta de la fábrica en el país de origen hasta la puerta de la planta en el país de destino. En otras palabras, no podía desarrollarse la producción fragmentada e integrada globalmente dado que los modos de transportes funcionaban de forma separada y desintegrada, por lo tanto, los flujos eran, en el mejor de los casos, de "puerto a puerto". Por lo mismo, los puertos tenían vínculos territoriales de menor alcance y una limitada acción estructurante en la organización del espacio (Martner, 1998).

A diferencia del periodo previo, el surgimiento del intermodalismo y la acción de los agentes sociales vinculados a las redes globales de transporte ha tenido repercusiones de gran relevancia. Los extensos ejes integrados de comunicaciones y transportes, que con el desarrollo del intermodalismo ha sido posible desplegar sobre el territorio, son una de las principales fuerzas estructuradoras del espacio en la actualidad. Por eso, los puertos donde ha penetrado con mayor fuerza la acción del intermodalismo han modificado notablemente sus conexiones espaciales y se convierten no sólo en nodos o "hubs" centrales de las redes productivas globales, sino también en nodos de articulación de regiones o fragmentos territoriales del interior.

Los puertos más dinámicos, al ampliar su vinculación territorial, invaden 
Carlos Martner Peyrelongue

y disputan la zona de influencia de puertos vecinos, otrora cautiva, debido a las limitaciones de accesibilidad, a las carencias de infraestructura y a la desarticulación de los sistemas de transporte. Por lo tanto, el intermodalismo y las mejoras físicas en la accesibilidad territorial propician la conformación de hinterlands comunes cada vez más competidos por los actores portuarios y por los operadores de transporte multimodal.

En este contexto, el puerto de la globalización, es decir, el puerto de la integración de redes, es el de los movimientos rápidos, el de la aceleración del tiempo, a diferencia del tiempo lento del puerto del proteccionismo. El puerto actualmente se revaloriza como espacio, como lugar diferenciado e incluso como región, en la medida en que se agilizan los movimientos, en que se acelera el tiempo.

Las posiciones neoclásicas e inclusive marxistas que tienden a concebir el espacio como distancia y, en ese tenor, hablan de la "anulación del espacio por el tiempo" (Harvey, 1989), son inadecuadas e insuficientes para pensar y analizar el carácter del puerto actual y el problema de su espacialidad. Por el contrario, en el afán de problematizar el tema, cabría señalar más bien que en el puerto de la globalización el tiempo revaloriza al espacio, le da contenido, forma y sustancia como lugar diferenciado que permite enlazar y, a la vez, marcar diferencias entre lo interno y lo externo.

\section{EL PUERTO Y LAS TRANSFOR- MACIONES ESPACIO-TEMPORALES DE LA GLOBALIZACIÓN}

\section{A. El puerto y la revalorización del espacio por el tiempo}

Con el predominio de los sistemas flexibles de producción y la posibilidad de fragmentar los procesos a nivel global, la circulación (de mercancías, valores e ideas) adquiere un papel fundamental en las relaciones económicas y sociales posfordistas. En este sentido, Milton Santos (1986) es enfático: "La aceleración de la circulación de bienes y personas se debe igualmente a las posibilidades abiertas por la aplicación de la ciencia a la producción. Las compañías transnacionales producen, cada vez con mayor frecuencia, partes de su producto final en diversos países y son, de este modo, un acelerador de la circulación. También gracias a estas empresas aumentó recientemente la necesidad de exportar e importar: una necesidad común a todos los países" (Santos, 1986: 39).

Ahora bien, tal aceleración de la circulación se debe precisamente al desdoblamiento de la producción inmediata sobre el espacio, propiciado por la fragmentación y externalización de procesos, cuya manifestación externa es la intensificación de flujos entre las diferentes fases y localizaciones. En este sentido, Castells (1994) concibe el concepto de "espacio de flujos" para caracterizar la tendencia emergente de organización del territorio en la fase de globalización de la economía-mundo. El "espacio de flujos”, y el concepto gemelo de territoriored desarrollado por Veltz (1994), se definen por las formas de conexión e integración entre fragmentos o nodos territoriales selectos y distantes a través del desarrollo de redes globales, en las cuales circulan flujos de todo tipo (mercancías, capitales, formas de gestión y organización, ideas, formas culturales, hábitos, etc.). El "espacio de flujos" o "territorio-red" se caracteriza por la fragmentación, la discontinuidad física y la separación espacio-temporal de los lugares o nodos que lo constituyen, a diferencia de las formas territoriales previas, como los territorios-zona (Veltz, 
El puerto y la vinculación entre lo local $\bar{y}$ lo global

1994), basados en la proximidad y en la contigüidad física. En otros términos, se trata de la constitución de nodos territoriales donde los actores sociales tienen la capacidad de articular simultáneamente, en tiempo real, procesos productivos y/o sociales fragmentados $\mathrm{y}$ físicamente separados a escala global. Ciertamente, esto propicia una selectividad de lugares privilegiados, frente a la exclusión de vastos ámbitos territoriales que no están integrados en las redes que, en la actualidad, apuntalan a la economía-mundo capitalista. Así, el "espacio de flujos", o el "territorio-red", es inconcebible sin el desarrollo tecnológico reciente en las comunicaciones y los transportes $\mathrm{y}$ sin las transformaciones que conducen a la externalización de la producción. ${ }^{3}$ Tales procesos han propiciado la intensificación de flujos y la conformación de redes de articulación territorial sobre el espacio global; redes que son a fin de cuentas el soporte físico, la condición sine qua non, de las formas territoriales emergentes.

Por lo tanto, con la intensificación de los flujos y aceleración de la circulación al interior de las vastas redes productivas globalizadas, el puerto que logra integrarse en esta dinámica entra en una relación espacio-temporal diferente a la del periodo previo. Al caracterizarse por ser un nodo principal de circulación de flujos (en especial de la circulación física de mercancías, pero también de la circulación de información y de formas tecnológicas y culturales), adquiere una importancia en la organización del terri-

\footnotetext{
${ }^{3}$ La externalización y el desdoblamiento de las cadenas productivas sobre amplios y distantes territorios debido a la globalización de la economía provoca que las actividades que anteriormente formaban parte de la circulación física, como el transporte, el almacenamiento y los flujos de información, ahora caigan dentro de la esfera de la producción inmediata (Martner, 1995).
}

torio que no tuvo en periodos anteriores, principalmente en América Latina, donde el esquema proteccionista, en buena medida, aisló a estos espacios limítrofes del resto de las regiones del país.

La aceleración de la circulación e intensificación de flujos está asociada a la aceleración del tiempo y de los movimientos. En este sentido, la ruptura obligada que supone el puerto tiende a reducirse al mínimo para mantener una relativa continuidad en la circulación de los flujos. Esta aceleración del tiempo y la forma específica en que se lleva a cabo en un lugar particular, es lo que otorga atributos y cualidades al puerto, revalorizándolo o devaluándolo como un espacio global viable dentro de la economíamundo. Aquí se podría discutir el hecho de que las instalaciones portuarias tienden a ser eslabonamientos estandarizados de las cadenas productivas globales y, en tanto tal, se convierten en "no-lugares", es decir, en lugares sin identidad propia, sin historia y sin posibilidad de ser apropiados socialmente. Desde esta perspectiva, las instalaciones portuarias no serían muy diferentes a otros "no lugares", mencionados por Marc Augé (1993), como los aeropuertos $\mathrm{y}$ los hoteles.

Pero el puerto como espacio singular, como ámbito local no puede reducirse a las instalaciones portuarias donde se realizan operaciones de carga, descarga y almacenamiento. El puerto incluye, entre otras cosas, lugares donde los diversos agentes sociales que están involucrados en su funcionamiento interactúan, zonas donde se localizan diferentes actividades productivas incluyendo el comercio y los servicios, además de los lugares de reproducción de la población local. Entonces, es el puerto en su conjunto, es decir, la ciudad portuaria o el "shipping district", el que 
Carlos Martner Peyrelongue

podría revalorizarse como lugar específico con características peculiares cuando el puerto logra conectarse con el tiempo de la globalización y la intensificación de flujos. ${ }^{4}$

Así pues, la ruptura se reduce en tiempo, los contactos son efimeros, las estadías menores y las características de enlace, de lugar de transferencia, de vínculo territorial con regiones interiores, son las que cobran mayor importancia en el puerto. Paradójicamente, en el puerto global, mientras menos prolongados son los contactos en ese espacio local, más se revaloriza el lugar, porque obviamente tal revalorización se produce en términos de eficiencia en el tratamiento de los flujos. En otras palabras, el puerto tiende a transformarse en nodo directo de valorización de las cadenas productivas globalizadas y, por lo tanto, en nodo de valorización del capital. En este contexto, la reducción de la estadía de los barcos, de la mercancía y de la tripulación produce contactos físicos menores, casi instantáneos; pero de la fugacidad de esos contactos depende, en gran parte, la capacidad de vinculación territorial y de articulación de redes del puerto actual.

La fugacidad del contacto físico que expresa, en última instancia, la aceleración del tiempo, se convierte en una medida de la capacidad de integración regional y global que tiene el puerto. ${ }^{5}$ Guizás una de las grandes diferencias del puerto de la globalización con el de épocas pretéritas es, precisamente, la

\footnotetext{
4 Podría plantearse la paradoja de que el "no lugar", o sea, las instalaciones portuarias, revaloricen al lugar, o sea, a la ciudad portuaria. Quizás existan otras actividades en las ciudades portuarias que reivindiquen el lugar, pero una forma específica de revalorizar el espacio-puerto es precisamente a través de la aceleración del tiempo.
}

fugacidad de los contactos físicos y sus implicaciones no sólo en el ámbito económico sino también en el cultural. En la década de los setenta, el tiempo de estadía promedio de un buque de carga en el puerto de Manzanillo era de 18 días, en 1996, cuando este lugar se había consolidado prácticamente como el único puerto del Pacífico mexicano integrado en las redes globales de producción-distribución, la estadía promedio de los buques de carga contenedorizada era sólo de 14 horas (Martner, 1998).

Por otra parte, si el puerto fue durante siglos un lugar primordial de contacto cultural, de entrada no sólo de mercancías (las cuales de por sí implican ya un contacto cultural), sino de valores e ideas de otros países, si durante mucho tiempo la presencia física tuvo un papel central en estos procesos, en la actualidad esa no parece ser una de sus características principales. Ahora, con el avance en la tecnología de telecomunicaciones existen otras formas de contacto cultural cuya penetración es directa e instantánea. La comunicación vía satélite, la televisión por cable, el intercambio electrónico de datos, el fax y las redes de computo conectadas a nivel internacional, son algunos de los elementos que permiten vinculaciones rápidas con culturas distantes.

Esa tecnología en telecomunicaciones, junto con aquella desarrollada en los transportes, ha contribuido a modificar notablemente la relación espacio-temporal en los puertos de este periodo de globalización de las relaciones económicosociales. Como se señaló antes, los

\footnotetext{
${ }^{5}$ Actualmente, los rendimientos en maniobras (medidos en tiempo) y la permanencia de embarcaciones (también medida en tiempo) son indicadores esenciales en la promoción del puerto. Así, en este nodo la aceleración del tiempo contribuye a la revalorización del lugar.
} 
El puerto y la vinculación entre lo local $\bar{y}$ lo global

contextos de la presencia en el puerto no guardan la posición indiscutible de antaño.

El desarrollo tecnológico permite a los agentes capitalistas globalizar operaciones, controlar procesos fragmentados en diversas localizaciones e impactar el desarrollo de espacios locales sin estar físicamente presente en todos ellos. Este proceso, al que Giddens llama "desanclaje" de las instituciones de la modernidad, "permitió la verificación de transacciones entre agentes ampliamente separados en tiempo y espacio" (Giddens, 1993:34). De esta forma, se logró una distanciación espacio-temporal ${ }^{6}$ nunca antes vista que ha sido definida bajo el concepto de espacio-tiempo simultáneo.

\section{B. El puerto y la simultaneidad espacio- temporal de la globalización}

El concepto de espacio-tiempo simultáneo (Hiernaux, 1995) ha sido definido como la posibilidad de incidir al mismo tiempo en procesos que se desarrollan en distintos lugares, por lo tanto, es factible la apropiación del espacio sin estar necesariamente en él. Los agentes capitalistas, apoyados en las innovaciones tecnológicas de las comunicaciones $\mathrm{y}$ transportes, están en posibilidad de controlar procesos fragmentados territorialmente y de influir, en tiempo real, en las actividades y el desarrollo de diversos espacios locales a partir de los espacios globales y sus redes.

\footnotetext{
${ }^{6}$ Según Giddens (1993), el "desanclaje" no es más que despegar las relaciones sociales de sus contextos locales de interacción para reestructurarlas en contextos espacio-temporales más amplios.
}

En el mismo sentido, Castells (1996) señala que con la informatización y el desarrollo de redes globales se modifica sustancialmente la relación espacio-tiempo. Ahora "el espacio determina al tiempo, con lo que se invierte una tendencia histórica"[...]. La idea de progreso, que durante los dos últimos siglos ha estado en los orígenes de nuestra cultura y nuestra sociedad, se basaba en el movimiento de la historia, de hecho, en la secuencia predeterminada de la historia guiada por la razón y por el impulso de las fuerzas productivas, escapando de las limitaciones de las sociedades y las culturas circunscritas al espacio. El dominio del tiempo y el control de la ritmicidad colonizaron territorios y transformaron el espacio en el vasto movimiento de la industrialización y la urbanización emprendido por el doble proceso histórico de la formación del capitalismo y el estatismo. Al convertirse en un ser estructurado, el tiempo conformó el espacio (Castells, 1996:500).

En cambio, ahora "la tendencia dominante en nuestra sociedad muestra la venganza histórica del espacio, al estructurar la temporalidad en lógicas diferentes, e incluso contradictorias, según la dinámica espacial. El espacio de flujos[...] disuelve el tiempo, desordenando la secuencia de los acontecimientos y haciéndolos simultáneos, con lo que se instala a la sociedad en una efimeridad eterna" (Castells, 1996:502)

Precisamente la simultaneidad espacio-temporal estructurada en las "global cities" y otros nodos territoriales con el apoyo de las nuevas tecnologias de comunicaciones y transportes, permite a los actores globales actuar en tiempo real en distintos fragmentos espaciales, aun sin estar físicamente en ellos, para co- 
Carlos Martner Peyrelongue

ordinar fases y procesos necesarios para la valorización del capital a escala global. ${ }^{7}$

Ahora bien, el puerto global, llamado también "hub" (Fossey, 1997), dado que concentra, procesa, integra y redistribuye los flujos de gran diversidad de cadenas productivas globales, se constituye en un nodo central de los medios de transporte y comunicación permeado por la concepción de espacio-tiempo simultáneo. Desde luego, esto no niega el hecho de que en el ámbito portuario coexistan otras determinaciones espacio-temporales correspondientes a periodos previos en el desarrollo de las fuerzas productivas locales; pero mientras más se integre el puerto a procesos capitalistas globalizados, mientras más habitantes de la localidad se vean involucrados en ellos, tanto directa como indirectamente, mayor será el dominio de una concepción de espacio-tiempo simultáneo sobre las actividades productivas y sociales de la localidad.

Las nuevas tecnologías y sistemas de información, que permiten concebir y articular en tiempo real procesos productivos ampliamente separados en tiempo y espacio, son la base de esta nueva concepción espacio-temporal. A través de los medios de comunicación se anuncian procesos que se desarrollan simultánea-

\footnotetext{
7 La simultaneidad espacio-temporal está estrechamente asociada a era de la fragmentación de la producción y a la incorporación de innovaciones técnicas y organizativas que permiten al capital desconcentrar fases productivas a grandes distancias y, a la vez, centralizar el control del proceso y de los beneficios. Los sistemas de producción "justo a tiempo" que valorizan el capital flexibilizando el proceso y reduciendo los inventarios en la planta requieren de una coordinación espacio-temporal, apoyada en medios de comunicación y transporte, que permita acceder a territorios específicos en tiempos exactos para no romper la continuidad de la producción.
}

mente en distintos lugares, pero que inciden y repercuten en el puerto gracias al papel unificador e integrador de las redes y los medios de transporte. El transporte se liga con el tiempo y funciona como medio para aunar el tiempo con el espacio, para enlazar la ausencia con la presencia, la instantaneidad con el aplazamiento. Así, el "desanclaje" de instituciones y agentes (Giddens, 1993), propio de la acumulación flexible, y el predominio de contextos de presencia y ausencia simultánea, permite integraciones territoriales más amplias.

La posibilidad tecnológica, que permite generar una concepción de espacio-tiempo simultáneo, hace viable la integración del espacio a larga distancia. De hecho, una característica del "espacio de flujos" o territorio de redes, que operan bajo esta lógica de simultaneidad, es la posibilidad de alcanzar incluso mayor integración con nodos territoriales alejados que con las propias localidades cercanas, ubicadas en la región o el país. Precisamente, el problema de esta forma de organización espacio-temporal es el alto grado de exclusión de los lugares que, independientemente de su cercanía o lejanía con respecto a los nodos globales, no logran articularse a las redes y flujos. En este sentido, se reduce la importancia de la distancia como forma de caracterización del espacio y lo que tiene que valorizarse ahora es la cualidad de la conexión e integración de los fragmentos espaciales selectos (nodos y "hubs") en una red territorial globalizada. El puerto, en esta perspectiva, puede convertirse en integrador de lugares y de tiempos. El puerto actual integra tiempos y espacios a través de la integración de los modos de transporte en una red global y, en este sentido, adquiere un rol relevante como articulador de fragmentos territoriales dispersos. 
El puerto y la vinculación entre lo local $\bar{y}$ lo global

Así, el espacio-tiempo simultáneo es precisamente una expresión clara de la articulación de lo local con lo global y el puerto aparece como un lugar privilegiado de confluencia de estos ámbitos, con sus características de nodo o "hub" de vinculación espacial y con las relaciones conflictivas que también se hacen presentes en su interacción dinámica.

\section{Puerto, REDES Y VINCULACIÓN LOCAL-GLOBAL}

\section{A. E1 puerto como articulador del ámbito local con el ámbito global}

El puerto de la globalización puede constituirse en nodo de integración territorial. Si se acepta este hecho, entonces cabría puntualizar que el puerto se fortalece por las vinculaciones que desarrolla principalmente hacia el interior. En ese sentido, tiene una doble función, por una parte, se integra a procesos globales desde su posición específica y diferenciada, es decir, como ámbito local, y, por otra, articula las regiones interiores con los procesos globales.

Así, este ente se reivindica como el lugar de enlace y articulación entre fragmentos territoriales interiores y el ámbito global, en la medida en que, a través de él, actores globales, como los Operadores de Transporte Multimodal, desarrollan redes de transporte integrado, "puerta a puerta", sin rupturas de carga. Por lo tanto, el fortalecimiento del puerto como lugar privilegiado en las relaciones globales lo convierte potencialmente en un elemento estructurado del territorio. Aparece como un potenciado de regiones en la medida en que la acción de los agentes sociales que operan en él, posibilite el despliegue de redes y corredores intermodales por determinados derroteros, pero al seleccionar tales senderos y concentrar flujos en corredores selectos se tiende, simultáneamente, a excluir a vastos territorios.

Desde luego, se está hablando del puerto de manera genérica. Por ejemplo, en países periféricos o semiperiféricos no todos los puertos y sus actores tendrán la misma posibilidad para articular el ámbito territorial local con el ámbito global. Como se mencionó previamente, el puerto no es sólo frontera física, también puede marcar una frontera tecnológica y cultural que dificulte la integración de ese ámbito local con el ámbito internacional.

En muchos puertos de los países periféricos difícilmente podrá acelerarse el tiempo y revalorizar la especificidad de estos entes como lugares dentro de un contexto de fragmentación productiva y acumulación flexible. Tal situación es probable si se considera que el territorio, en un momento dado, tiene la capacidad, como señala Milton Santos (1990), de condicionar la evolución de otras estructuras sociales.

La existencia de configuraciones previas en la región donde está implantado el puerto puede implicar relaciones débiles con los procesos productivos globales y, por lo tanto, la acción articuladora del puerto y sus actores se verá reducida o anulada. Después de todo, algunos procesos se adaptan a las formas espaciales preexistentes mientras que otros requieren crear nuevas formas para insertarse en ellas (Santos, 1990: 160). En efecto, hay lugares que sufren un desfase difícil de remontar, cuando se trata de insertarse en procesos globales. De hecho, tal situación es bastante frecuente en las regiones y puertos de los países periféricos y conduce a la exclusión de vastos territorios de las dinámicas dominantes en la actualidad. 
Carlos Martner Peyrelongue

En todo caso, lo que se pretende señalar con estas observaciones es que el puerto tendrá efectos mayores en la configuración territorial de una región dependiendo, entre otras cosas, de la acción de agentes sociales específicos que posibilitan la extensión del hinterland portuario y del fortalecimiento del propio puerto como entidad local capaz de singularizarse $\mathrm{y}$, a la vez, de integrarse en procesos de largo alcance.

Ahora bien, definir hasta qué punto el ámbito local se interrelaciona desde una sólida posición con el ámbito global o, por el contrario, sólo se subordina a este último, es un aspecto que ha generado una amplia discusión. En la actualidad este debate se ha producido, sobre todo, en la geografía económica. Allí "se ha replanteado la cuestión del ámbito local y de la región a partir de las nuevas tendencias de la economía mundial". (Hiernaux, 1995). Las posiciones se han polarizado entre aquellos que otorgan un papel central y privilegiado al ámbito local y los que, por el contrario, ubican a esta instancia en un lugar subordinado.

Por una parte, aquellos investigadores que resaltan la emergencia de ámbitos regionales y, en particular, de distritos industriales ${ }^{8}$ ponen el acento en la conformación de densos tejidos locales de relaciones económicas y sociales que se sustentan en elementos como una nueva cultura empresarial vinculada al lugar, en la creación de redes intrarregionales que posibilitan la competencia, pero también la cooperación entre agentes locales para enfrentar a los ámbitos productivos externos.

${ }^{8}$ Entre éstos destacan los trabajos de Poire y Sabel (1984) y también los de Storper y Harrison (1994).
En el polo opuesto, autores como Amin y Robins plantean que la producción descentralizada y fragmentada está espacialmente vinculada por redes integradas a escala mundial, las cuales son supervisadas principalmente por un pequeño número de gigantescas sociedades anónimas. Por lo tanto, "la economía local sólo se puede ver como un nodo de la red económica mundial y puede no tener existencia significativa alguna fuera de este contexto" (Amin y Robins, 1991:219).

Lo que parece evidente es que no todos los "locales" tienen la misma posibilidad de fortalecerse internamente y constituirse en sólidas estructuras regionales. Por lo tanto, en una red de relaciones globales cabe esperar lugares dominantes y nodos subordinados, principalmente en países periféricos. Habría que considerar también que la división internacional del trabajo dificulta, en regiones periféricas, la consolidación de ámbitos locales fuertes. En todo caso, tanto la mistificación de lo local como el desdén por este ámbito parecen posiciones extremas en una gama de posibilidades que se define, como se comentó previamente, más por la compleja interacción del nexo local-global que por la acción aislada en cada uno de estos ámbitos.

\section{B. Las redes en la vinculación local-global}

Independientemente del resultado a que pueda conducir la discusión entre los apologistas de los distritos industriales y aquellas corrientes que ponen énfasis en los circuitos monopólicos internacionales de acumulación, es importante resaltar los principales elementos que pueden contribuir al análisis territorial del puerto actual.

114 eure 
El puerto y la vinculación entre lo local $\bar{y}$ lo global

Resulta notable el hecho de que ambas posiciones destacan, aunque de manera diferente, el papel de las redes en la configuración de relaciones económicas, sociales y territoriales de la actualidad. En efecto, con la fragmentación de la producción y la consecuente externalización de funciones las empresas han tenido que crear redes para poder coordinar los procesos que se ejecutan en plantas ubicadas en diferentes lugares. El desarrollo tecnológico reciente ha favorecido la configuración de redes y las ha hecho extensivas a otras esferas de la vida social. Actualmente hay redes educativas, redes de organizaciones políticas y sociales, redes de grupos étnicos, etcétera.

Los autores que reivindican los distritos industriales, como Zurla, no dejan de poner énfasis en la importancia de la estructura reticular para la configuración y "funciojerárquico" (Zurla, 1991:161).

Evidentemente, las redes aquí encuentran su máxima expresión en el ámbito local. Ello no invalida el hecho de que investigadores que defienden esta línea, como Storper (1991), expliciten que el distrito industrial no está reñido con las relaciones interindustriales a larga distancia, ni con la presencia de grandes empresas en el ámbito local. Storper dice que las relaciones a larga distancia "no impiden, de modo alguno, a una localidad ser un distrito industrial". (Storper, 1991:246). Por lo tanto, reconoce la existencia de redes extensas que vinculan los distritos locales con el mundo, aunque enfatiza el papel del ámbito local en estas relaciones globalizadas.

Por el contrario, para Amin y Robins, tienden a predominar las redes extensas que rebasan ampliamente el ámbito local y en las cuales las grandes empresas multinacionales imponen su domi- nio (Amin y Robins, 1991). Castells, más cerca de esta ultima postura, hace evidente la dependencia del ámbito local frente al ámbito global. Aunque no desdeña la importancia y fuerza que han adquirido algunas sociedades locales, establece claramente el dominio de los imperativos de la economía global capitalista. Para este autor, en las redes de articulación espacial, configuradas en la fase de globalización, "ningún lugar existe por sí mismo, desde que los lugares son definidos por los flujos". En este sentido, acepta que las redes tienen un papel fundamental en la configuración espacial emergente, denominada "espacio de flujos”, y tal rol muchas veces va en detrimento de la importancia de lugares o ámbitos locales específicos. "Los lugares no desaparecen pero sus lógicas y sus significados llegan a ser absorbidos en la red" (Castells, 1996:412). Así, "los procesos dominantes de nuestras sociedades son articulados en redes que conectan diferentes lugares y asignan a cada uno de ellos un rol y un peso en la jerarquía de la generación de riqueza, el procesamiento de información y el manejo del poder, lo cual, en última instancia, condiciona el destino de cada lugar" (Castells, 1996:415).

En definitiva, el "espacio de flujos" o territorio de redes, que tiende a predominar en la actualidad, está basado en redes físicas y electrónicas, pero esas redes conectan lugares o nodos específicos, con características sociales, culturales y físicas bien definidas (Castells, 1996). El tipo de red más fácil de visualizar como representativa del espacio de flujos está constituida por el sistema de toma de decisiones de la economía global, particularmente por el sistema financiero global. El trabajo de Sassen (1991) sobre las ciudades globales ("global cities") es ejemplificador en este sentido. En él se muestra el rol fundamental de las llamadas ciudades globales 
Carlos Martner Peyrelongue

como sitios de conducción de la economía global y la dependencia de las sociedades locales frente a las funciones direccionales localizadas en aquéllas. "El análisis de las ciudades globales proporciona la ilustración más directa de los centros que organizan el espacio de flujos en nodos y hubs, pero esta lógica no está limitada para un solo medio de capital de flujos" (Castells, 1996:415).

En efecto, "más allá de las 'global cities', otros continentes, naciones y economias regionales tienen sus propios nodos (y hubs) que se conectan con las redes globales. Cada uno de esos nodos requiere una adecuada infraestructura tecnológica, un sistema auxiliar de empresas que suministren servicios de soporte, un mercado de trabajo especializado y un sistema de servicios atendido por fuerza de trabajo profesional" (Castells, 1996:413).

Por lo tanto, las redes que conforman el "espacio de flujos" castelliano no son de un solo tipo. Estas pueden corresponder tanto a la actividad financiera como a otras expresiones del capital. Por ejemplo, la producción industrial fragmentada requiere ligas entre operaciones ubicadas en diferentes lugares, por lo tanto, los flujos de productos y de información entre firmas circulan por determinados nodos y hubs de la red global, entre los que destacan los puertos globales. Estos nodos y hubs tienen también diferentes características. En algunos predominan fases complejas del proceso de circulación del capital-dinero, en otros, como los hubs portuarios, son más significativas las fases de produccióndistribución del capital-mercancía, de la gestión de inventarios e información.

Ahora bien, seguramente los hubs portuarios vinculados a redes tenderán a disolver fronteras tecnológicas y organizacionales, suavizarán las fronte- ras físicas (mediante el aprovechamiento de la tecnología y la incorporación de capital fijo), pero más difícil será anular la frontera cultural de la identidad regional, sobre todo si este ámbito busca reforzar su posición como lugar específico dotado de características particulares. De todas formas la identidad local o regional en los ámbitos portuarios no puede ser concebida como instancia pura e incólume ante las influencias externas. Por el contrario, en el puerto de la globalización es claro que "lo que estructura lo local no es simplemente eso que está en escena, sino que la 'forma visible' de lo local encubre las distantes relaciones que determinan su naturaleza" (Giddens, 1993). Precisamente, lo que permite aunar lo local con lo global, en formas que hubieran resultado impensables en sociedades locales tradicionales, es el "desanclaje" de las modernas instituciones capitalistas (Giddens, 1993), las cuales, mediante vinculaciones de larga distancia facilitadas por el vertiginoso desarrollo tecnológico, expresado en la constitución de redes, pueden influir en una región determinada, sin estar presente físicamente todo el tiempo.

En el planteamiento de Castells las características y necesidades de las redes, junto con los agentes sociales que las construyen y representan, son los elementos que definen en gran medida la posición de los ámbitos locales (los cuales, más que lugares son conceptualizados como nodos o hubs). Así, "la función que debe ser cumplida por cada red define las características de los lugares que llegan a ser sus nodos privilegiados" (Castells, 1996:414). En efecto, "La teoría del espacio de flujos comienza con el reconocimiento implícito de que las sociedades están organizadas asimétricamente en torno a los intereses dominantes específicos de cada estructura social" [...] "Pero tal dominación no es puramente estructural. Es propicia- 
El puerto y la vinculación entre lo local $\overline{\text { lo global }}$

da, sin duda concebida, decidida e implementada por actores sociales". (Castells, 1996:415.)

Por lo tanto, los nodos de dirección, los lugares de producción y los hubs de comunicaciones y transportes "son definidos a lo largo de la red y articulados en una lógica común por las tecnologías de la comunicación, por el desarrollo de la informática, por las bases microelectrónicas de datos y por la producción flexible" (Castells, 1996:414). Según Castells, la infraestructura tecnológica que construye las redes define la nueva estructura espacial. "Esa infraestructura tecnológica es ella misma la expresión de las redes de flujos cuya arquitectura y contenido es determinado por el poder que domina en nuestro [sistema] mundo" (Castells, 1996:413).

Lo cierto es que la formación de redes, tanto económicas como sociales y culturales, ejerce una notable influencia en la organización territorial actual. La emergencia del espacio de flujos o del territorrio-red (Veltz, 1994) puede implicar condicionamientos fuertes e inclusive la desestructuración de ámbitos locales específicos, sobre todo en la periferia y semiperiferia del sistema-mundo capitalista, donde difícilmente logran consolidarse nodos privilegiados de las redes globales. Sin embargo, en ámbitos locales hegemónicos como las "global cities" de Sassen (1991), en los distritos industriales de los países centrales (Storper y Harrison, 1994) o en los hubs portuarios estas nuevas formas territoriales pueden ser un elemento relevante en la afirmación de tales espacios, si es que las redes globales realmente fortalecen los tejidos y las relaciones locales, cuestión que no ha sido plenamente demostrada.

\author{
V. CONClusión: EL PUERTO COMO \\ NODO ARTICULADOR DEL ESPACIO \\ DE FLUJOS.
}

Los puertos articulados en red tienden a constituirse en nodos o hubs de concentración, integración, procesamiento y distribución de flujos a través de redes físicas que movilizan capital-mercancía e información. Como espacio frontera, tienen la posibilidad de estructurar encadenamientos y redes productivas globalizadas hacia los territorios interiores. A diferencia de otros nodos o hubs, tienen la propiedad de articular tanto su propio espacio local, así como los fragmentos interiores del espacio nación o del espacio continental de pertenencia, con las redes globales.

En este sentido, la concepción previa de la geografía económica de los litorales que ubicaba al puerto como la parte central de una tríada espacial que incluye también el "hinterland" y el "foreland" (Bird, 1971) tiende a ser insuficiente para explicar la forma de inserción territorial que adquieren los puertos en la actualidad. Con el desarrollo de redes intermodales, integradas de puerta a puerta, la separación en dos nítidos estancos diferenciados y etiquetados, como el foreland o zona de conexión externa (de ultramar) y el hinterland o zona de influencia interna (de tierra adentro), representa una falsa dicotomía (Charlier, 1992). En efecto, con la supresión de la ruptura de carga en puertos debido al desarrollo de tecnologías como la del contenedor, el hinterland y el foreland se vuelven variables interdependientes, las cuales no pueden ser separadas y, por lo tanto, los flujos que se mueven entre estos ámbitos deben ser analizados como un continuum. En otras palabras, en el análisis espacial de la vinculación del puerto el segmento de tierra adentro de los flujos debe ser analizado como una porción de un flujo con- 
Carlos Martner Peyrelongue

tinuo dentro de una red de produccióndistribución que es articulada y procesada por nodos o hubs portuarios, más que como un flujo entre el puerto y su zona de influencia natural o cautiva, pregonado en el modelo tradicional del hinterland.

Precisamente, uno de los cambios fundamentales en las características de los vínculos espaciales de los puertos es la desarticulación de los hinterlands cautivos que estaban fundados en elementos como la cercanía geográfica o la identidad común con el entorno regional más próximo. En efecto, con el desarrollo de redes de producción-distribución integradas por sistemas intermodales de transporte a través de hubs portuarios selectos, los hinterlands dejan de ser zonas de influencia cautiva y se convierten en ámbitos espaciales de competencia o disputa entre muchos puertos. De esta forma, los puertos en red tienden a amplificar y multiplicar sus zonas de influencia territorial, penetrando y desestructurando los hinterlands de los puertos regionales menos integrados, de tal manera que estos últimos entran en fases de estancamiento y decadencia. Por ejemplo, recientemente los puertos norteamericanos del Pacífico (Long Beach y Los Angeles) han extendido en miles de kilómetros sus segmentos intermodales de tierra adentro (inland), hasta captar incluso los flujos de comercio exterior de muchos estados y ciudades mexicanas (incluyendo a la Ciudad de México), de esta forma han desestructurado los hinterland y los vínculos territoriales de numerosos puertos nacionales que, desde fines de la década de los ochenta, muestran signos de estancamiento. Sólo un puerto del litoral del Pacífico mexicano (Manzanillo) parece haber logrado insertarse en redes globales articuladas por sistemas intermodales de transporte y esto le ha permitido, simultáneamente, expandir sus vínculos con múltiples regiones del país y competir por los flujos de carga nacional con los puertos norteamericanos.

Lo cierto es que el puerto, concebido como un hub o nodo del espacio de flujos, ya no importa tanto por sí mismo, sino por el vínculo y por la integración que alcance con otros nodos o fragmentos espaciales de "tierra adentro" (inland). Si la reestructuración del sistema capitalista mundial supone una selectividad de lugares ${ }^{9} \mathrm{y}$, por lo tanto, "no requiere el control del espacio continuo [...] para operar" (Hiernaux, 1993) entonces inevitablemente se requerirá de redes, nodos y hubs articuladores de ese espacio discontinuo. En este contexto, el puerto no es un nodo simple que vincula un lugar con otro, sino un nodo peculiar que ejerce atracción sobre múltiples redes y, a la vez, produce una articulación múltiple de ámbitos territoriales desiguales y distantes. En buena medida, desde la perspectiva espacial, el rasgo preponderante del puerto está ahora más determinado por la articulación e integración a larga distancia que establece con otros nodos territoriales de la red global que por las relaciones o interacciones con su propio ámbito local. Sin embargo, esto no excluye el hecho de que algunos hubs portuarios, principalmente de países centrales, logren establecer densos tejidos de relaciones con su ámbito local inmediato.

En definitiva, el puerto de la globalización es una estructura compleja $\mathrm{y}$ dinámica que no encuentra una caracterización única y permanente; sin embargo, está íntimamente ligado a las determinaciones espacio-temporales propias de la actual fase de desarrollo del

\footnotetext{
${ }^{9}$ Por ese proceso es que, en buena medida, el nexo local-global está en el centro del debate territorial actual.
} 
El puerto y la vinculación entre lo local y lo global

sistema-mundo. Genéricamente, el puerto aparece ahora como un lugar o nodo privilegiado para la emergencia e instauración de la nueva forma de organización del territorio basada en la existencia de redes. En efecto, sus características como nodo o hub articulador de espacios y tiempos lo ubican como un elemento que potencia el desarrollo del espacio de flujos y del territorio-red, con todas las implicaciones que esto tiene sobre la selectividad y exclusión de vastos territorios, principalmente en los países periféricos.

\section{BIBLIOGRAFIA}

Amin, A. y Robins, K. (1991). "Distritos industriales y desarrollo regional: Limites y posibilidades". En Sociología del Trabajo. Número especial ¿Neofordismo o especialización flexible?, España.

Augé, M. (1993). Los 'no lugares'. Espacios del anonimato, una antropología de la sobremodernidad. Editorial Gedisa. Barcelona, España.

Benko, G. y Dunford, M. (1992). "Changement structurel et organisation spatiale du système productif". Revue $d^{\prime}$ Economie Régionale et Urbaine $\mathrm{N}^{ }$ 2. París, Francia.

Bird, J. (1971). "The gateway concept". En Seaports an seaport terminals. Hutchinson University Library, London.

Castells, M. (1994). "European cities, the informational society and the global economy". New Left Review N ${ }^{\circ} 204$, pp. 18-32. London.

Castells, M. (1996). "The rise of the network society". The information age: Economy, society and culture, Volume I., Blackwell Publishers Ltd. Oxford, Gran Bretaña.

Charlier, J. (1992). "Ports and hinterland connections". En Dolman, A. y Van Ettinger, J. (Comps.) Ports as nodal points in a global transport system. Pergamon Press and International Ocean Institute. Great Britain.

Fossey, J. (1997). "Relay gold". Revista Containerisation International, Vol. 30, $\mathrm{N}^{\circ}$ 6, junio de 1997. Emap Business Communications, New Jersey, U.S.A.

Giddens, A. (1993) Consecuencias de la modernidad. Ed. Alianza Universidad. $\mathrm{N}^{\circ}$ 760. Madrid, España.

Giddens, A. (1988) "Time, Space an Regionalisation”. En Derek, G. y Urry, J. (Comps.) Social relations and spatial structures. Ed. MacMillan. London.

Harvey, D. (1989). The condition of Posmodernity. Cambridge. Basil Blackwell. U.S.A.

Hiernaux, D. (1993). "Globalización, integración y nuevas dimensiones territoriales: una aproximación conceptual”. Mimeografiado. México.

Hiernaux, D. (1995). "La región insoslayable”. Revista Eure, Vol. XXI, N 63. Instituto de Estudios Urbanos. PUC. Santiago de Chile, Junio.

Lipietz, A. y Leborgne, D. (1990). "Nuevas tecnologías, nuevas formas de regulación: algunas consecuencias espaciales". En Alburquerque, F., De Mattos, C. y Jordán, R. Revolución tecnológica y reestructuración productiva: Impactos y desafios territoriales, Grupo Editor Latinoamericano, ILPES y IEU. Buenos Aires, Argentina.

Martner, C. (1995). "Innovación tecnológica y fragmentación territorial". Revista EURE, Vol XXI, $\mathrm{N}^{\circ} 63$, p. 69-76, junio de 1995. IEU-PUC, Santiago de Chile.

Martner, C. (1998). "La apertura comercial y los puertos del Pacífico en la reestructuración del territorio en México". Tesis Doctoral. Doctorado en Ciencias Sociales, Area de concentración en Sociedad y Territorio. División de Ciencias Sociales y Humanidades, Universidad Autónoma MetropolitanaXochimilco, México, D.F. 
Carlos Martner Peyrelongue

Piore, M. y Sabel, C. E. (1984). "The second industrial divide". Basic Book. New York, U.S.A.

Ramírez, B. (Comp.) (1991) Nuevas tendencias en el análisis regional. Ed. UAM-Xochimilco. México.

Santos, M. (1986). "Espacio y Método". Rev. Geo Critica. Septiembre, 1986. Universidad de Barcelona. España.

Santos, M. (1990) Por una geografía nueva. Ed. Espasa Calpe. Colec. Espasa Universidad. España.

Sassen, S. (1991) The global city. New York, London, Tokyo. Princeton University Press. New Jersey, USA.

Silva, V. (1993) "El interés de los distritos industriales para la formulación de una política regional". Mimeografiado. Instituto de Estudios Urbanos. Universidad Católica de Chile. Santiago.

Storper, M. (1991) "Tres respuestas a Ash Amin y Kevin Robins”. En Sociología del Trabajo. Número especial ¿Neofordismo o especialización flexible? España.

Storper, M. y Harrison, B. (1994). "Flexibilidad, jerarquía y desarrollo regional: Los cambios de estructura de los sistemas productivos industriales $\mathrm{y}$ sus nuevas formas de articulación del poder en los años 90". En Benko y Lipietz (Comp.) Las regiones que ga- nan. Distritos y redes: los nuevos paradigmas de la geografía económica, Edicions Alfons El Magnánim. Generalitat Valenciana. Diputació Provincial de Valéncia. España.

Unctad (1992). "La comercialización del puerto y las perspectivas del puerto de tercera generación". Informe de la Secretaría de la UNCTAD. Comisión del Transporte Marítimo. Grupo Intergubernamental Especial de Expertos en Puertos. Naciones Unidas.

Veltz, P. (1994). "Jerarquía y redes en la organización de la producción y el territorio". pp. 281-300. En Benko y Lipietz (Comp.) Las regiones que ganan. Distritos y redes: los nuevos paradigmas de la geografía económica. Edicions Alfons El Magnánim. Generalitat Valenciana. Diputació Provincial de Valéncia. España.

Wallerstein, I. (1990). "El análisis de los sistemas mundiales". En La teoría social, hoy. Compilado por Giddens y Turner. Ed. CNCA-Alianza Editorial. México.

Wallerstein, I. (1996). Después del Liberalismo. Editorial Siglo XXI, México.

Zurla, P. (1991) "Nuevas tecnologías, estrategias de redes y economías locales”. En Sociología del Trabajo. Número especial ¿Neofordismo o especialización flexible? España. 